\title{
Conservative Surgery in the Management of Adenomyosis
}

\author{
Masato Nishida*, Hiroya Itagaki, Yasuo Otsubo, Yuko Arai \\ Department of Obstetrics and Gynecology, National Hospital Organization, Kasumigaura Medical Center, Tsuchiura, Japan \\ Email address: \\ nishidamasato@mac.com(M. Nishida),itacyoko1986@gmail.com(H. Itagaki),tubo@nms.ac.jp(Y. Otsubo),yarai@dia-net.ne.jp(Y. Arai) \\ ${ }^{*}$ Corresponding author
}

\section{To cite this article:}

Masato Nishida, Hiroya Itagaki, Yasuo Otsubo, Yuko Arai. Conservative Surgery in the Management of Adenomyosis. Journal of Gynecology and Obstetrics. Vol. 7, No. 1, 2019, pp. 8-16. doi: 10.11648/j.jgo.20190701.12

Received: December 29, 2018; Accepted: February 11, 2019; Published: February 28, 2019

\begin{abstract}
Since 2002, we have performed adenomyomectomy for 1780 women with uterine adenomyosis. We classified adenomyosis in these patients as focal $(n=1313)$, diffuse $(n=450)$ or cystic $(n=17)$ type according to the distribution of the focuses seen in magnetic resonance imaging (MRI) findings. Three different surgical methods for focal, diffuse and cystic adenomyosis are utilized at our institution. The adenomyosis lesion is excised using a loop electrode of a high-frequency cutter in all methods. The median resected lesion weight of focal, diffuse and cystic adenomyosis were $94 \mathrm{~g}(1-1156 \mathrm{~g}), 150 \mathrm{~g}(10-1595 \mathrm{~g})$, and $16 \mathrm{~g}$ (3-45 g), respectively. The mean visual analogue score for dysmenorrhea decreased from 9.1 to 1.0 and heavy menstrual bleeding was improved in all cases. Following the procedure, 370 pregnancies occurred in 294 patients, of which 153 (41.9\%) were the result of natural conception. Pregnancy rate of the patients younger than 40 years were $35.1 \%$ in focal type, $25.4 \%$ in diffuse type and $88.9 \%$ in cystic type. Among those patients, we experienced 5 cases of uterine rupture. Of 1462 patients who underwent surgery more than 2 years prior to the time of writing, recurrence occurred in $150(10.3 \%)$. Our findings indicate that conservative surgery for uterine adenomyosis using a high-frequency resection device is effective for both focal and diffuse type.
\end{abstract}

Keywords: Adenomyosis, Conservative Surgery, Classification, Prognosis, Pregnancy, Recurrence

\section{Introduction}

Recently, conservative management for uterine disease has become an important issue in Japan, because the numbers of women who marry or achieve pregnancy at a later age have been increasing. Traditionally, adenomyosis has been treated with a hysterectomy. However, affected women who wished to preserve their uterus, because of future plans for marriage or becoming pregnant, were forced to endure the severe dysmenorrhea and menorrhagia, severely reducing their quality of life.

The purpose of an adenomyomectomy procedure is to improve the quality of life for the women who wish to preserve their reproductive capability. Although good reviews of the procedure have been presented [1], those reports included only a small number of subjects [2-28], with none examining more than 1000 patients. Here, we present details of 1780 patients who have received an adenomyomectomy at our hospital since 2002 .

\section{Adenomyosis: Classification, Conservative Surgery and Prognosis}

\subsection{Histogenesis Associated with Adenomyosis}

Since the first report of the condition by Cullen in 1908 [29], uterine adenomyosis has been considered to arise from an invasion of normal endometrium tissue into the myometrium. In 2012, Kishi et al. [30] proposed that adenomyosis can be differentiated into 3 subtypes according to MRI findings; that developed from the endometrium (subtype I), from the endometriosis (subtype II), and from metaplastic changes of the Mullerian system (subtype III). We have confirmed that classification, though consider subtype III to be most closely related with cystic adenomyosis [31].

\subsection{Classification of Adenomyosis}

For uterus removal, it is not necessary to locate the focus, though it is very important to know that location in patients undergoing conservative surgery. A preoperative diagnosis 
should be made based on T2-weighted MRI imaging with sagittal, transverse and oblique view perpendicular to the uterine axis. In typical cases, trans-vaginal ultrasonography can also be used for diagnosis of adenomyosis, while MRI findings are necessary when conservative surgery is planned.

We classify adenomyosis into focal, diffuse and cystic type according to the distribution of the focuses show in MRI findings [31]. The diffuse type is defined as adenomyosis occupying both the anterior and posterior walls, with connection between those (Figure 1A). An adenomyosis lesion containing bloody fluid in the center of the focus is designated as the cystic type (Figure 1B), while that other than diffuse or cystic type occupying a part of uterine wall is categorized as focal type. Even though adenomyosis generally arises along the midline of the uterus, most cases of cystic adenomyosis arise in younger ages and as solitary lesions on the lateral wall [31]. Focal adenomyosis is classified into anterior wall (Figure 2A), posterior wall (Figure 2B), fundal (Figure 2C), and lateral wall (Figure 2D), according to the location of the focuses. Cases with focuses that are distributed between the anterior wall and fundus (Figure 3A) or posterior and lateral walls (Figure 3B) are also considered as focal type. Diffuse type adenomyosis can be further classified into 2 subtypes, i.e., complete (Figure 4A), which is defined as tissue completely replaced by adenomyosis, and incomplete (Figure 4B), in which normal uterine muscle partially remains. Adenomyosis lesions that are round and clearly demarcated from surrounding normal muscle are designated as nodular type (Figure 4C), and considered to be included in the subtype III (intramural type) classification of Kishi et al [30]. However, we think that this type has an unclear meaning, because nodular adenomyosis coexists with other types of adenomyosis in many cases [31].

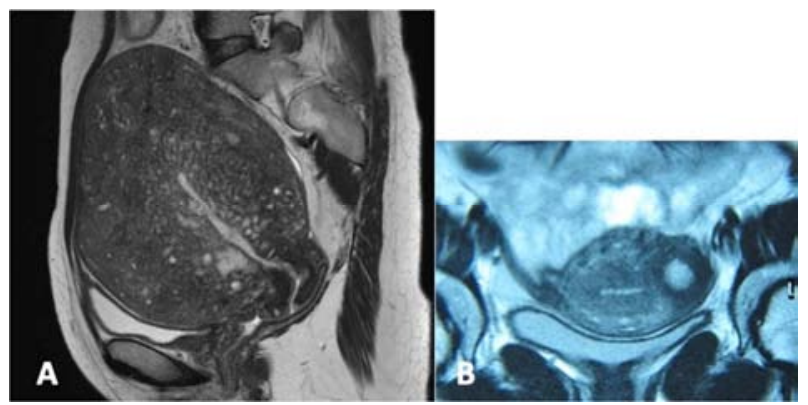

Figure 1. T2-weighted MR imaging, A sagittal view of diffuse type adenomyosis. $B$ colonal view of cystic type adenomyosis.

As shown in figure 1, the poorly demarcated low-intensity portion shows adenomyosis completely involving both the anterior and posterior areas of the uterine wall. Adenomyosis can been seen solery in the lateral portion of the uterine wall. The lesion is surrounded by intact muscular structures.

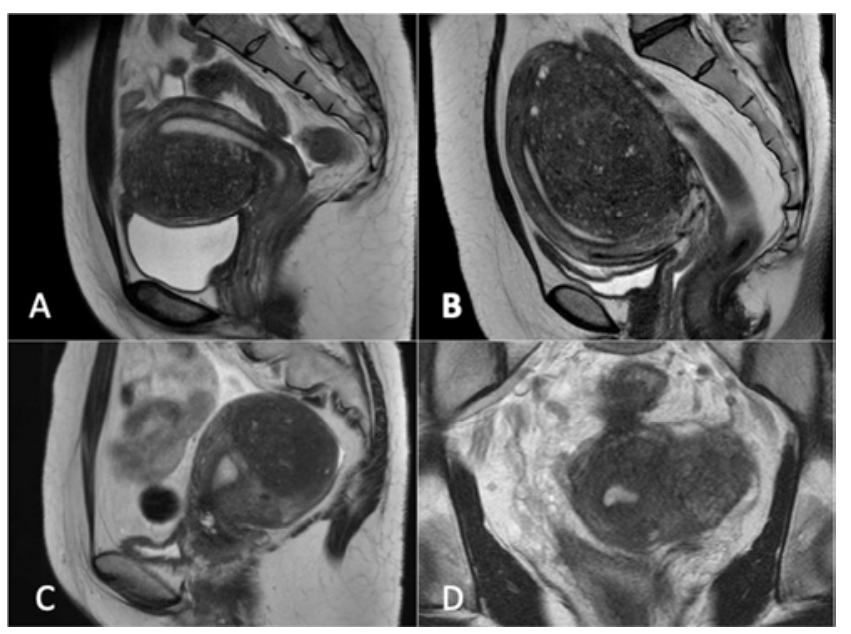

Figure 2. T2-weighted MR imaging sagittal view. A. sagittal view of anterior focal type adenomyosis. B. sagittal view of posterior focal type adenomyosis. C. sagittal view of fundal focal type adenomyosis. D. oblique view of left lateral focal type adenomyosis.

In figure $2 \mathrm{~A}-\mathrm{C}$, adenomyosis can be seen in the anterior portion of the uterine wall, posterior portion of the uterine wall and fundal portion of the uterine wall. In figure $2 \mathrm{D}$, adenomyosis can be seen in the left lateral portion of the uterine wall.
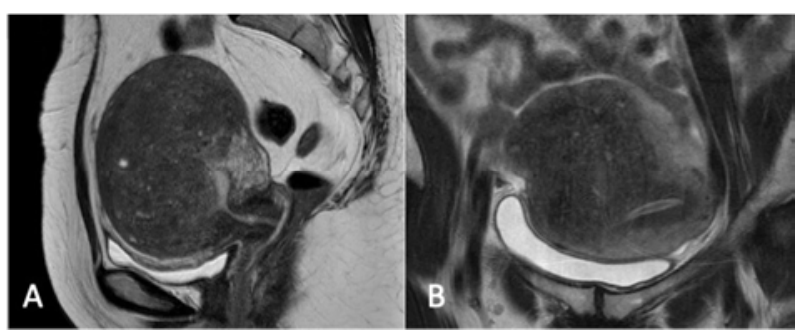

Figure 3. T2-weighted MR imaging. A. sagittal view of uterus. B. oblique view of uterus.

As shown in figure 3A, adenomyosis can be seen in both the anterior and fundal portion of the uterine wall, and was classified as focal type adenomyosis. figure $3 \mathrm{~B}$, adenomyosis can be seen in both the posterior and lateral portions of the uterine wall, and was classified as focal type.

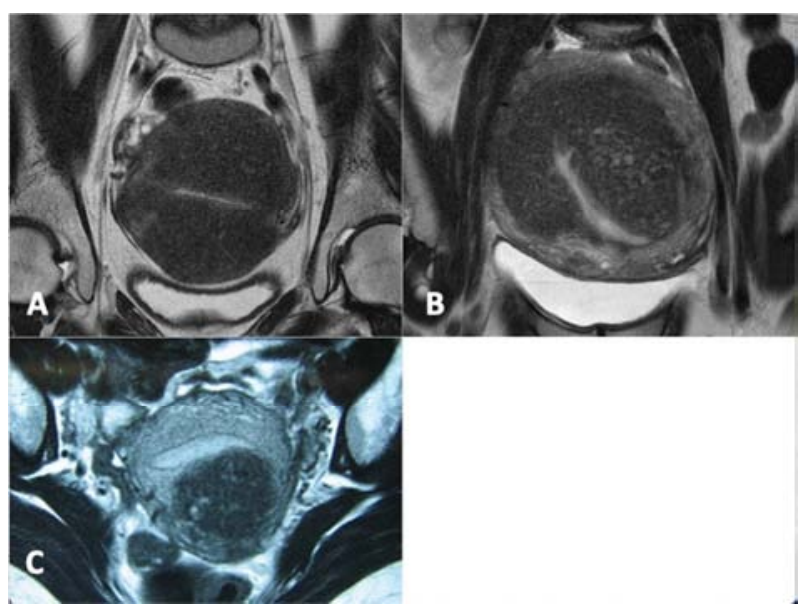

Figure 4. T2-weighted MR imaging $A \& B$. oblique view of diffuse type adenomyosis. C. oblique view of nodular type adenomyosis. 
In figure 4A, adenomyosis can be seen throughout the uterine wall, and was classified as complete diffuse type adenomyosis.In Figure 4B, adenomyosis can be seen on both the anterior and posterior uterine walls, with connection those areas, while normal uterine muscle is shown on the anterior and left lateral uterine walls. This was classified as incomplete diffuse type adenomyosis.In Figure 4C. Adenomyosis can be seen exclusively on the posterior uterine wall, with the lesion surrounded by intact muscular structures.

The distribution of different types of adenomyosis and their clinical profiles are shown in Table 1. Forty-six patients whose
MRI findings were not available were excluded. Additionally, 4 patients with posterior wall type and 9 with diffuse type were also excluded because they consisted of both subtype I and II. Subtype I was found to develop at every site, including the anterior wall in $28.4 \%$, posterior wall in $25.9 \%$, and diffuse type in $43.1 \%$ of our cases. Conversely, most cases of subtype II cases developed in the posterior wall (anterior wall in 5.3\%, posterior wall in $91.5 \%$, diffuse type in $0.2 \%$ ). Subtype III mainly developed in cystic adenomyosis cases, such as in the anterior wall in $4.3 \%$, and posterior wall in $21.7 \%$, as well as nodular type in $8.7 \%$, and cystic type in $65.2 \%$ (Table 1 ).

Table 1. Patients characteristics according to subtypes.

\begin{tabular}{|c|c|c|c|c|}
\hline & I: intrinsic $(n=735)$ & II: extrinsic $(n=603)$ & III: intramural $(n=23)$ & Entirely $(n=360)$ \\
\hline \multicolumn{5}{|l|}{ Classification } \\
\hline Anterior $(\mathrm{n}=308), \mathrm{n}(\%)$ & $209(28.4)$ & $32(5.3)$ & $1(4.3)$ & $66(18.3)$ \\
\hline Posterior $(n=907), n(\%)$ & $190(25.9)$ & $552(91.5)$ & $5(21.7)$ & $160(44.4)$ \\
\hline Nodular $(\mathrm{n}=15), \mathrm{n}(\%)$ & $7(1.0)$ & $6(1.0)$ & $2(8.7)$ & $0(0)$ \\
\hline Fundus $(\mathrm{n}=27), \mathrm{n}(\%)$ & $6(0.8)$ & $9(1.5)$ & $0(0)$ & $12(3.3)$ \\
\hline Lateral $(\mathrm{n}=12), \mathrm{n}(\%)$ & $6(0.8)$ & $3(0.5)$ & $0(0)$ & $3(0.8)$ \\
\hline Cystic (n=15), n (\%) & $0(0)$ & $0(0)$ & $15(65.2)$ & $0(0)$ \\
\hline \multicolumn{5}{|l|}{ Reproductive History } \\
\hline Nulligravid (n=897), (\%) & $245(33.3)$ & $435(72.1)$ & $18(78.3)$ & $199(55.3)$ \\
\hline Multigravid $(n=638),(\%)$ & $365(49.7)$ & $121(20.1)$ & $2(8.7)$ & $150(41.7)$ \\
\hline Multiparous $(n=245),(\%)$ & $125(17.0)$ & $47(7.8)$ & $3(13.0)$ & $70(19.4)$ \\
\hline \multicolumn{5}{|l|}{ Coexisting Disease } \\
\hline Myoma $(n=926)$ & $368(50.1)$ & $360(59.7)$ & $8(34.7)$ & $190(52.8)$ \\
\hline Endometriosis $(\mathrm{n}=592)$ & $73(9.9)$ & $387(64.2)$ & $3(13.0)$ & $129(35.8)$ \\
\hline
\end{tabular}

\subsection{Procedure for Conservative Surgery}

\subsubsection{Method to Distinguish the Focus from Normal Muscle and Instruments for Resection}

Adenomyosis lesions show invasion of the surrounding normal uterine muscle without a clear borderline. Nevertheless, they can be distinguished with palpation, because normal uterine muscle is elastic soft, while the adenomyosis focus is relatively firm.

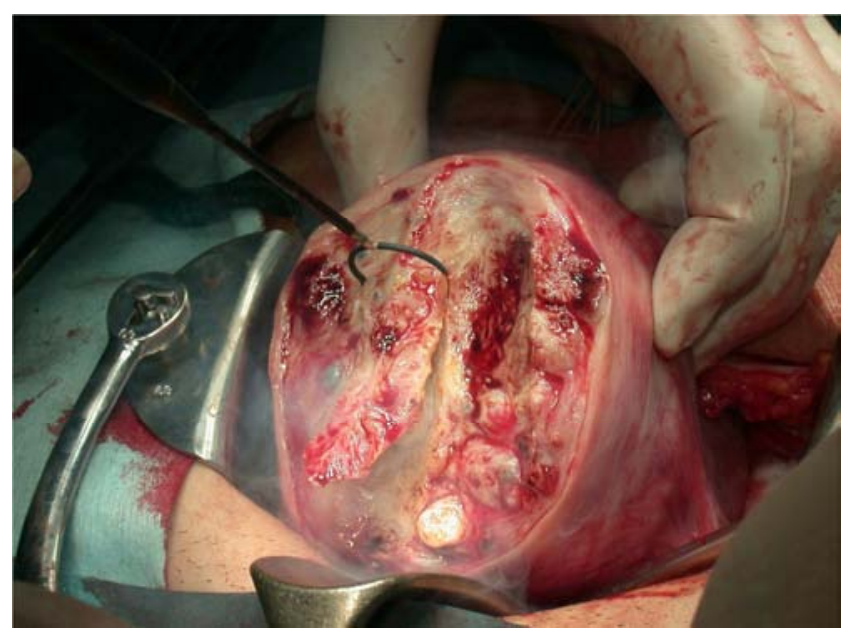

Figure 5. Electrical resection of adenomyosis lesion. The focus was scooped from the uterine wall using a high-intensity surgical loop.
For electrical resection, we use a high-intensity surgical loop (Figure 5) (loop-type electrode; Honest Medical, Tokyo, Japan) at $124 \mathrm{~W}$. As compared to normal surgery, which can be likened to cutting a steak with a knife and fork, an adenomyomectomy is similar to eating ice cream with a spoon, with the focus scooped out from the uterine wall using a high-intensity surgical loop.

\subsubsection{Surgical Procedures}

\section{i. Adenomyomectomy for focal adenomyosis}

The surgical procedures available can divided into 3 categories; type I for focal adenomyosis, type II for diffuse adenomyosis, and the classical method for cystic and nodular adenomyosis. At the beginning of our experience with these cases, we excised the adenomyosis lesion through the surface serosa using a high-frequency loop electrode without opening the uterine cavity. However, we later noticed a high rate of recurrence associated with this procedure, because part of the focus remained. We subsequently made some modifications, including opening the uterine cavity from the focus side and resecting the surface serosa along with the adenomyosis lesion. This modified procedure resulted in a lower rate of recurrence and higher rate of pregnancy, though we noticed that uterine rupture occasionally occurred during pregnancy. We consider that a post-adenomyomectomy uterine rupture is due to the placenta percreta induced by villous infiltration along to the uterine suture site [32]. Our present technique includes opening the uterine cavity from the opposite side of the focus (a so-called back window wound), insertion of a finger into 
the uterine cavity, measuring the thickness between the focus and uterine cavity, and guiding the loop electrode to excise the adenomyosis lesion. At the back window, the wound is closed in 2 layers with a synthetic absorbable suture, with the first layer was closed using an interrupted suture, and the second layer using a continuous in and out suture. Thereafter, the wound is closed as a monolayer with an interrupted suture on the focus side.

\section{ii.Adenomyomectomy for diffuse adenomyosis}

For the diffuse type, following longitudinal and asymmetrical dissection of the uterus with a high-frequency electrical surgical knife (spear-type electrode; Honest Medical, Tokyo, Japan) at $124 \mathrm{~W}$, the myometrium is diagonally dissected as if hollowing out the uterine cavity, then the adenomyosis lesion is excised from the incision area site using a loop electrode, after which the uterus is rejoined [20]. At the beginning of our experience with these cases, a transverse incision was made on the fundus to allow insertion of a finger into the cavity [20]. However, we noticed that a uterine rupture during pregnancy could occur in association with this procedure [32], thus changed from a transverse incision to a longitudinal incision in the middle of the fundus (so-called center vent wound), then perform closure twice with the outer side myometrial layers.

iii.Adenomyomectomy for cystic adenomyosis

It is not necessary to open the uterine cavity for treating cystic adenomyosis, as the adenomyosis lesion can be resected completely including the central cystic portion from the surface serosa without opening the cavity. We term this a classical procedure, which can also be adopted for nodular adenomyosis.

\section{iv. Adhesiolysis for periuterine adhesions}

Most of the patients with subtype II adenomyosis have severe pelvic adhesions, which should be disrupted prior to an adenomyomectomy. Although this type of adhesiolysis is very difficult to treat, pelvic adhesions in patients with subtype II adenomyosis are regular and it is possible to perform adhesiolysis easily with an understanding of this regularity. The types of adhesion can be divided into 2 categories. The first is an adhesion between the posterior uterine wall and intestine, which is characterized as beginning from a very thin adhesion in the surface area and then gradually changing into dense adhesion in the deep portion. This type of adhesion tends to be thick and strong, thus adhesiolysis must be carefully performed along the uterine surface wall so as to not injure the intestine. The other type features the posterior uterine body strongly flexed and fixed to the back wall of the cervix (Figure 6). This adhesion tends to occupy a large portion of the uterine posterior surface, though is relatively weak, so the adhered portions can be separated by rubbing strongly along the uterine surface with a finger. The most important point to consider when performing an adhesiolysis procedures in both types is to accurately locate the layer to be separated and the site of the internal os of the uterus. Bimanual palpation from both the posterior and anterior sides is helpful to locate the uterine internal os.

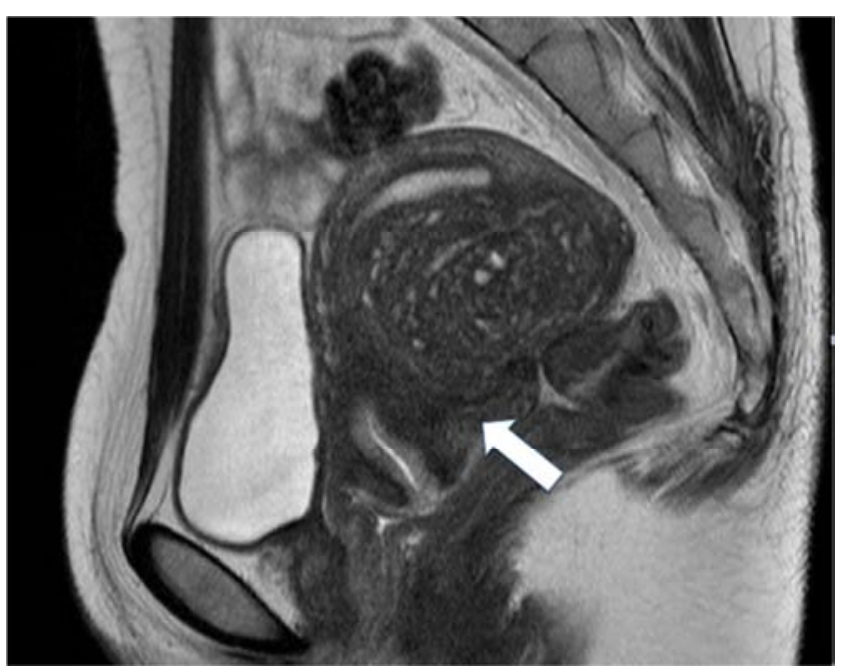

Figure 6. Adhesion between uterine body and cervix. T2-weighted MR imaging, sagittal view, of subtype II adenomyosis showing posterior uterine body strongly flexed and fixed to the back wall of the cervix (arrow), with appearance of fetal deflexion.

\subsubsection{Other Surgical Procedures Performed Along with Adenomyomectomy}

With an adenomyomectomy, we performed a myomectomy in $47.4 \%(843 / 1780)$, adhesiolysis in $45.4 \%(809 / 1780)$, enucleation for an ovarian chocolate cyst in $26.0 \%(462 / 1780)$, metroplasty for a uterine anomaly in $1.6 \%(28 / 1780)$, and enucleation for adenomatoid tumor in $1.8 \%(32 / 1780)$ of our patients.

\subsection{Patient Characteristics}

Conservative surgery for adenomyosis is the surgery which respects a hope of the patient and self-decision. Therefore, all who want to preserve their uterus are accepted. For those who do not wish for a future pregnancy, we recommend a hysterectomy. Ultimately, the feelings of the patient are respected and all who desire to preserve their uterus have been accepted. Excluded from this study were 6 patients diagnosed with malignant tumors, including ovarian cancer $(n=2)$, endometrial stromal sarcoma $(\mathrm{n}=2)$, and leiomyosarcoma $(n=2)$, and 12 patients not pathologically diagnosed with adenomyosis, except for leiomyoma or adenomatoid tumor. All patients were managed using the same clinical procedure for open abdominal surgery for benign disease cases at our hospital. The normal 11-day period of hospitalization is the same as that for patients undergoing a leiomyomectomy or abdominal total hysterectomy.

\subsubsection{Age Distribution}

The age of our patients ranged from 14 to 52 years, with a mean of 38.7 years. Age distribution every 5years is shown in Figure 7. Although the age distribution of focal and diffuse adenomyosis had a single peak in the late $30 \mathrm{~s}$, that of cystic adenomyosis showed peak in both the 20s and 30s. This finding may indicate 2 different origins for the histogenesis of cystic adenomyosis. 
(\%)

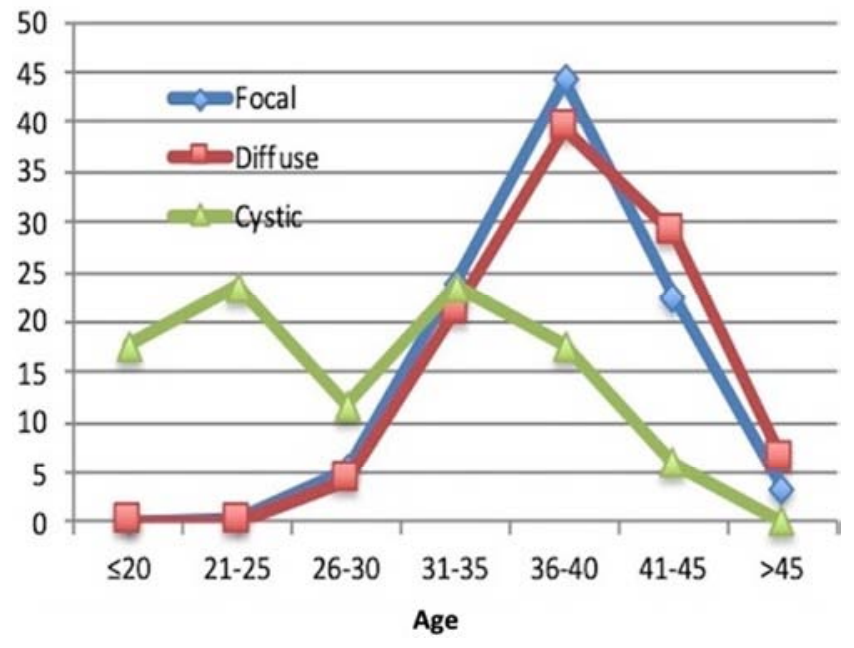

Figure 7. Age distribution of patients with 3 types of adenomyosis.

\subsubsection{Marriage History and a Hope for Pregnancy}

At the time of surgery, the number of married patients including remarriage and fact marriage totaled 1208 (67.9\%), while that of unmarried including widows and after divorce totaled $513(28.8 \%)$. Following surgery, 60 of the unmarried patients became married and 16 of the married patients divorced. For all patients, 83.4\% (1484/1780) hoped for pregnancy in the future, while $5.6 \%(100 / 1780)$ did not have and $5.7 \%(102 / 1780)$ replied that they were undecided. When classified by age, $79.7 \%(51 / 64)$ of patients under 30 years old, $86.6 \%$ (923/1066) aged 30-39, and 78.5\% (510/650) aged 40 or over expressed a desire for future pregnancy. In addition, $91.2 \%(895 / 981)$ of married patients had no living child.

\subsubsection{Reproductive History}

Among all patients, 50.4\% (897/1780) were nulligravid, $35.8 \%(638 / 1780)$ were multigravid, and $13.8 \%(245 / 1780)$ were multiparous. As for reproductive history and classification of adenomyosis, the percentage of nulligravid, multigravid, and multiparous patients with subtype I was $33.3 \%$ (245/735), 49.7\% (365/735), and 17.0\% (125/735), respectively, with subtype II was $72.1 \%$ (435/603), 20.1\% (121/603), 7.8\% (47/603), respectively, and with subtype III was $78.3 \%(18 / 23), 8.7 \%(2 / 23), 13.0 \%$ (3/23), respectively (Table 1). Thus, multigravid patients were more frequently subtype I, while nulligravid patients were more frequently classified as subtype II or III. These findings may suggest that pregnancy can induce some of the subtype I adenomyosis and that subtype II adenomyosis is accompanied by endometriosis.

\subsubsection{Coexistent Diseases}

Coexisting leiomyoma was seen in 52.0\% (926/1780) of all patients with adenomyosis and in 50.1\% (368/735) classified as subtype I, 59.7\% (360/603) classified as subtype II, and $34.7 \%(8 / 23)$ classified as subtype III. In all patients with adenomyosis, coexisting endometriosis was seen in $33.3 \%$ (592/1780), including9.9\% (73/735) classified as subtype I, $64.2 \%(387 / 603)$ classified as subtype II, and 13.0\% (3/23) classified as subtype III (Table 1). In regard to the location of adenomyosis, endometriosis was seen in $17.8 \%$ (56/315) with anterior adenomyosis, 49.6\% (466/940) with posterior adenomyosis, and $11.6 \%(52 / 450)$ with diffuse type. Thus, coexistent endometriosis was significantly more frequent in type II adenomyosis and posterior wall adenomyosis cases. On the other hand, we encountered 4 patients with bladder endometriosis, of whom 3 had coexisting anterior subtype II adenomyosis and 1 coexisting anterior entire invasion type. At total of 31 of our patients had anterior subtype II adenomyosis, in whom bladder endometriosis was coexistent in $3(9.7 \%)$ (Figure 8), and all of those patients had severe adhesions between the uterine anterior wall and bladder. Uterine anomalies were found in 28 patients, each of whom underwent a concomitant metroplasty procedure.

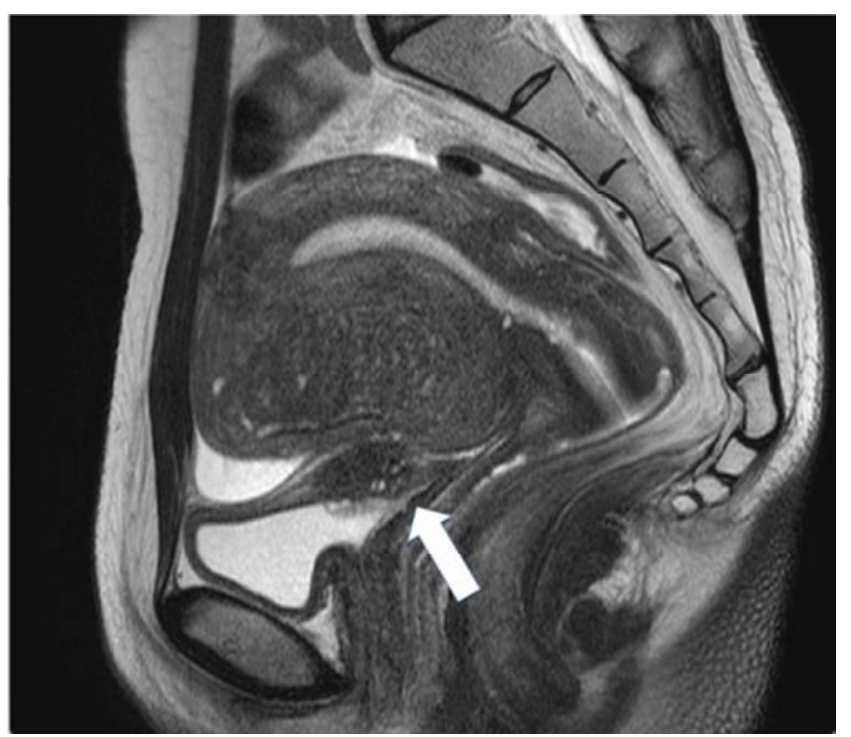

Figure 8. T2-weighted MR imaging, sagittal view, of relatively rare anterior uterine wall subtype II adenomyosis. This type occasionally coexists with bladder endometriosis (arrow).

\subsubsection{Past History}

Four hundred ninety-five patients $(27.8 \%)$ had a history of previous surgery, including 243 (13.7\%) who underwent open abdominal surgery for gynecologic diseases and $189(10.6 \%)$ who had laparoscopic surgery. Additionally, 193 (10.8\%) had received surgery for endometriosis and 94 (5.3\%) a myomectomy. gonadotropin-releasing hormone analogue (Gn-RHa), dienogest, and low-dose estrogen progestin (LEP) therapy were previously performed in $300(16.9 \%), 106$ $(6.0 \%)$, and $79(4.4 \%)$, respectively.

\subsubsection{History of Adenomyomectomy}

The patients included 90 who experience recurrence following an adenomyomectomy, performed at our hospital for 31 and at other hospitals for 59. Seven patients conceived and 4 had viable births following an adenomyomectomy, with uterine ruptures encountered during pregnancy in 2 of those cases. Ten patients had recurrence after other types of operations.

\subsection{Results and Prognoses}

All of the patients were examined at 2 weeks after discharge 
and found to be performing daily life activities. Dysmenorrhea and menorrhagia were evaluated at 3 months after surgery, with MRI performed for all patients and pregnancy permitted. Generally, these patients were followed annually without treatment until menopause.

\subsubsection{Resected Lesion Weight}

The weights of the resected lesions in focal adenomyosis cases ranged from 1 to $1156 \mathrm{~g}$, with a median value of $94 \mathrm{~g}$, from 10 to $1595 \mathrm{~g}$ in diffuse type cases, with a median value of $150 \mathrm{~g}$, and from 3 to $45 \mathrm{~g}$ in cystic type cases, with a median value of $16 \mathrm{~g}$ (Table 2).

\subsubsection{Operative Time}

The median operative time for focal adenomyosis was 132 minutes (53-510 minutes), while that was 174 minutes (87-651 minutes) for diffuse adenomyosis and 74 minutes (43-110 minutes) for cystic adenomyosis (Table 2).

\subsubsection{Blood Loss}

Median blood loss was $288 \mathrm{~g}(1-3799 \mathrm{~g})$ in focal adenomyosis, $415 \mathrm{~g}(10-8921 \mathrm{~g})$ in diffuse adenomyosis, and $47 \mathrm{~g}$ (1-216 g) in cystic adenomyosis cases (Table 2).

Table 2. Operative Results.

\begin{tabular}{llll}
\hline & Focal $(\mathbf{n = 1 3 1 3})$ & Diffuse $(\mathbf{n}=\mathbf{4 5 0})$ & Cystic $(\mathbf{n}=\mathbf{1 7})$ \\
\hline Median Operative Time (min) (Range) & $132(53-510)$ & $174(87-651)$ \\
Median Resected Lesion weight (g) (Range) & $94(1-1156)$ & $150(10-1595)$ & $74(43-110)$ \\
Median Blood Loss (g) (Range) & $288(1-3799)$ & $415(10-8921)$ \\
\hline
\end{tabular}

\subsubsection{Complications During and After Surgery}

Intestinal injury occurred in 4 patients with severe fixation following a previous surgery, while bladder injury occurred in 2 and ureter injury in 1 . All of the patients underwent a repair surgical procedure and none required a stoma. In 1 patient whose cervix was removed with the adenomyosis lesion, the uterine body was sutured to the vagina. No postoperative complications, including uterine necrosis, uterine abscess, or severe infection, were noted. Three patients with a uterine muscle hematoma and 1 with a uterine muscle infection were treated conservatively. None of the patients had uterus removal during or after surgery, except in cases of recurrence. Seventeen patients had sub-ileus or ileus after surgery, and all but 1 were treated conservatively. Asherman's syndrome developed in 2 patients after surgery, with menstruation after adhesiolysis confirmed based on hysteroscope findings in 1 patient, while the other was followed according to her wish because she underwent a second surgery (Table 3). During follow-up after surgery, endometriosis was found to have developed in the abdominal wound in 4 patients.

Table 3. Complications.

\begin{tabular}{ll}
\hline None & 1747 \\
Ileus & 17 \\
Ileus managed surgery & 1 \\
Injury to intestinally tract & 4 \\
Infection & 3 \\
Uterine wall hematoma & 3 \\
Injury to bladder & 2 \\
Asherman's syndrome & 2 \\
Injury to ureter & 1 \\
\hline
\end{tabular}

\subsubsection{Dysmenorrhea}

In our patients, dysmenorrhea was greatly improved after surgery (Figure 9). Soon after the operation, the mean visual analogue score for dysmenorrhea decreased from $9.15 \pm 1.43$ to $1.05 \pm 1.58$ in the focal adenomyosis cases, from $9.08 \pm 1.61$ to $1.02 \pm 1.56$ in the diffuse adenomyosis, and from $9.73 \pm 0.55$ to $1.30 \pm 1.99$ in cystic adenomyosis cases. Furthermore, at 5 years after surgery, that score for dysmenorrhea remained low at $2.37 \pm 2.46$ in the focal, $2.62 \pm 2.52$ in the diffuse, and $1.22 \pm 1.38$ in the cystic cases. Comparison performed using Student's t-test revealed a significant difference between VAS value before and after surgery.

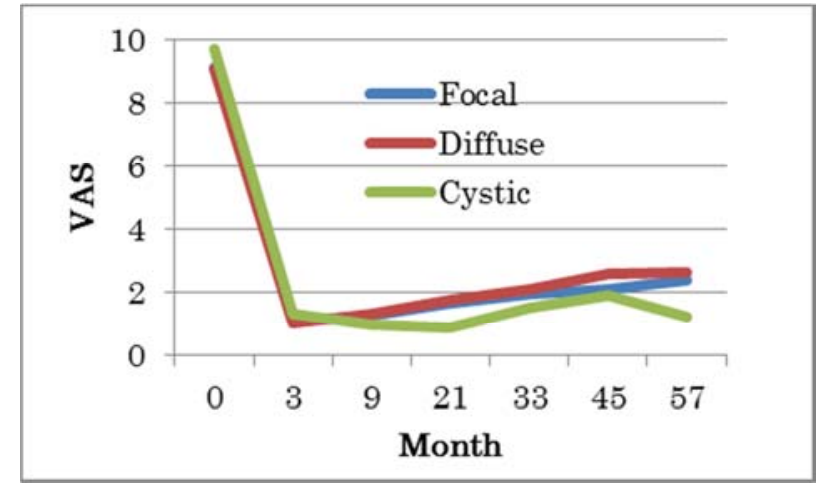

Figure 9. Images showing dysmenorrhea before and after surgery.

\subsubsection{Menorrhagia}

The mean blood hemoglobin concentration in the untreated patients was $7.1 \pm 2.0 \mathrm{~g} / \mathrm{dl}$, while that in those who underwent surgery was $12.5 \pm 1.4$ and $13.2 \pm 1.4 \mathrm{~g} / \mathrm{dl}$ at 3 months and 5 years, respectively, after the operation. Most patients suffered from iron deficiency anemia before the procedure, with conservative surgery improving menorrhagia more than dysmenorrhea.

\section{Reproductive Outcome and Recurrence After Conservative Surgery}

\subsection{Pregnancy Outcome After Surgery}

Following surgery, 370 pregnancies occurred in 294 patients, with term deliveries in 155 , preterm deliveries in 41 , and 25 patients presently pregnant. A total of 196 healthy babies have been successfully delivered (Table 4). With 1 pregnancy considered to represent 1 patient (term delivery $>$ 
preterm delivery $>$ pregnant $>$ abortion), 231 patients with focal adenomyosis, 54 with diffuse adenomyosis, and 9 with cystic adenomyosis became pregnant, of whom 153, 28, and 6, respectively, gave birth to viable infants (Table 5). Post-operative pregnancy rates for the 1087 married patients at more than 1 year after surgery and for patients who hoped for pregnancy are shown according to age in Table 6 . The rate of pregnancy was $59.5 \%$ for patients in their $20 \mathrm{~s}, 33.6 \%$ for those in their early $30 \mathrm{~s}, 31.5 \%$ for those in their late $30 \mathrm{~s}, 11 \%$ for those in their early $40 \mathrm{~s}$, and $3.9 \%$ for those in their late $40 \mathrm{~s}$.

Table 4. Outcome of Pregnancy after Surgery.

\begin{tabular}{llll}
\hline & Focal $(\mathbf{n}=\mathbf{2 8 7})$ & Diffuse $(\mathbf{n = 7 3})$ & Cystic $(\mathbf{n = 1 0})$ \\
\hline Term Delivery $(\mathrm{n}=155)$ & 132 & 17 & 6 \\
Preterm Delivery $(\mathrm{n}=41)$ & 28 & 13 & 0 \\
Abortion $(\mathrm{n}=95)$ & 65 & 28 & 2 \\
Chemical Abortion $(\mathrm{n}=38)$ & 4 & 9 & 0 \\
Ectopic Pregnancy $(\mathrm{n}=7)$ & 2 & 3 & 0 \\
Hydatidiform Mole $(\mathrm{n}=3)$ & 6 & 0 & 1 \\
Induced Abortion $(\mathrm{n}=6)$ & 21 & 0 & 0 \\
Pregnant $(\mathrm{n}=25)$ & & 3 & 1 \\
\hline
\end{tabular}

Table 5. Outcome of Pregnancy after Surgery (One pregnancy represents 1 patient).

\begin{tabular}{|c|c|c|c|}
\hline & Focal $(n=231)$ & Diffuse $(n=54)$ & Cystic $(n=9)$ \\
\hline Term Delivery $(\mathrm{n}=149)$ & 127 & 16 & 6 \\
\hline Preterm Delivery $(n=38)$ & 26 & 12 & 0 \\
\hline Abortion $(n=54)$ & 38 & 15 & 1 \\
\hline Chemical Abortion $(n=19)$ & 13 & 6 & 0 \\
\hline Ectopic Pregnancy $(n=7)$ & 4 & 3 & 0 \\
\hline Hydatidiform Mole $(n=2)$ & 1 & 0 & 1 \\
\hline Induced Abortion $(n=4)$ & 4 & 0 & 0 \\
\hline
\end{tabular}

Table 6. Pregnancy rate after surgery according to age at surgery

\begin{tabular}{ll}
\hline Age & Pregnancy (\%) \\
\hline$<30(\mathrm{n}=37)$ & $22(59.5)$ \\
$30-34(\mathrm{n}=214)$ & $72(33.6)$ \\
$35-39(\mathrm{n}=485)$ & $153(31.5)$ \\
$40-44(\mathrm{n}=300)$ & $33(11.0)$ \\
$\geq 45(\mathrm{n}=51)$ & $2(3.9)$ \\
\hline
\end{tabular}

On the other hand, regarding pregnancy rate and classification of adenomyosis, the pregnancy rate in focal adenomyosis cases was $27.3 \% \quad(221 / 810)$, diffuse adenomyosis cases was $19.5 \% \quad(52 / 267)$, and cystic adenomyosis cases was $90 \%$ (9/10) (Table 7). Among patients who were younger than 40 years at the time of surgery, those pregnancy rates were $35.1 \%$ (196/558), 25.4\% (43/169), and $88.9 \%(8 / 9)$, respectively (Table 7$)$. Thus, the postoperative pregnancy rate was highest in patients treated for cystic adenomyosis.

Table 7. Pregnancy rate after surgery According to Classification.

\begin{tabular}{lll}
\hline & Total $(\%)$ & Under 40 years old $(\%)$ \\
\hline Focal $(\mathrm{n}=810)$ & $221(27.3)$ & $196(35.1)$ \\
Diffuse $(\mathrm{n}=267)$ & $52(19.5)$ & $43(25.4)$ \\
Cystic $(\mathrm{n}=10)$ & $9(90.0)$ & $8(88.9)$ \\
\hline
\end{tabular}

In 356 patients who successfully achieved pregnancy, excluding 5 unclear cases, an artificial reproductive technique (ART) was used in $212(58.1 \%)$ and natural pregnancy occurred in $153(41.9 \%)$ (Table 8$)$. When these results were evaluated from the perspective of type of adenomyosis, the rate of natural pregnancy was $56.5 \%$ $(78 / 138)$ in subtype I, $26.4 \%(32 / 121)$ in subtype II, and $100 \%$
$(10 / 10)$ in cases of cystic adenomyosis (Table 8$)$, while ART pregnancy rates were $43.5 \%(60 / 138), 73.6 \%(89 / 121)$, and $0 \%$, respectively (Table 8). Following surgery, all pregnancies in cystic adenomyosis cases and more than half in subtype I were natural. On the other hand, more than $70 \%$ in subtype II were ART. There was a clear difference in achievement of pregnancy after surgery between subtype I and subtype II. Comparisons were performed using a chisquared test, which revealed a significant difference between natural pregnancy rates for the type I and type II adenomyosis cases. The rate of abortion was more frequent in diffuse type, occurring in 50.7\% (37/73), as compared with $32.8 \%(94 / 287)$ in focal type and $20 \%(2 / 10)$ in cystic type cases (Table 4$)$.

Table 8. Treatment of Pregnancy after surgery.

\begin{tabular}{lll}
\hline Classification & ART (\%) & Natural (\%) \\
\hline Subtype I $(\mathrm{n}=138)$ & $60(43.5)$ & $78(56.5)$ \\
Subtype II $(\mathrm{n}=121)$ & $89(73.6)$ & $32(26.4)$ \\
Focal $(\mathrm{n}=287)$ & $170(60.3)$ & $112(39.7)$ \\
Diffuse $(\mathrm{n}=73)$ & $42(57.5)$ & $31(42.5)$ \\
Cystic $(\mathrm{n}=10)$ & 0 & $10(100)$ \\
\hline
\end{tabular}

\subsection{Measures to Improve Pregnancy Outcome}

A uterine rupture during pregnancy occurred in 5 cases, 3 with focal and 2 with diffuse type. That is induced by placenta percreta when implantation occurs on the uterine scar at the focus where the uterine cavity was opened. A uterine rupture after surgery causes sudden abdominal pain without uterine contractions when intra-abdominal bleeding starts from the placenta percreta site [32]. We have improved our surgical procedure and do not open the 
uterine cavity from the focus side, but rather from the opposite side of the uterine wall. In cases of diffuse adenomyosis, we have also modified the procedure and changed from a transverse large incision of the uterine fundus to a longitudinal small incision, then cover the site twice with the outer myometrial layer.

\subsection{Recurrence After Surgery}

Based on a definition of recurrence that includes a continuous elevation of serum CA125, reappearance of dysmenorrhea, and detection of adenomyosis lesions in MRI findings, 150 of 1462 patients were diagnosed with recurrence more than 2 years after surgery. The recurrence rate was $12.3 \%$ $(45 / 367)$ in diffuse adenomyosis, 9.6\% (104/1080) in focal adenomyosis, and $6.7 \%(1 / 15)$ in cystic adenomyosis cases (Table 9). Among patients with focal adenomyosis, the recurrence rate of those with subtype I was $11.5 \%$ (37/322), higher than that of subtype II at $6.6 \%$ (32/482) (Table 9).

Table 9. Recurrence Rate.

\begin{tabular}{ll}
\hline Classification & Recurrence (\%) \\
\hline Subtype I $(\mathrm{n}=322)$ & $37(11.5)$ \\
Subtype II $(\mathrm{n}=482)$ & $32(6.6)$ \\
Focal $(\mathrm{n}=1080)$ & $104(9.6)$ \\
Diffuse $(\mathrm{n}=367)$ & $45(12.3)$ \\
Cystic $(\mathrm{n}=15)$ & $1(6.7)$ \\
\hline
\end{tabular}

We consider that subtype I adenomyosis is likely to recur on the wall opposite of the initial focus, which we term de novo recurrence (Figure 10) and noted in 67.6\% (25/37) of affected patients. In patients with coexisting endometriosis or leiomyoma, the rate of recurrence after surgery was $18.4 \%$ (90/488) and 9.5\% (69/728), respectively.

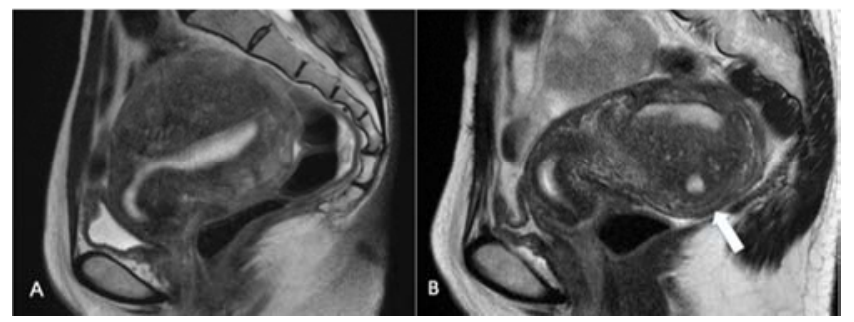

Figure 10. MRI findings of de novo recurrence. T2-weighted MR images, sagittal section, obtained $(A)$ before and $(B) 5$ years after surgery.

The adenomyosis lesion in this case arose on the opposite side of the uterine wall (arrow) and the original focus disappeared.

\subsection{Treatment for the Recurrence}

For most of the patients with recurrence, medical therapy such as treatment with dienogest has been adopted, though a re-adenomyomectomy was indicated in 31 and a hysterectomy performed in 20 patients. In 7 patients, medical treatment for recurrence was concluded because of menopause. Among patients diagnosed with recurrence, 28 later became pregnant and 11 delivered healthy babies.

\section{Conclusion}

Adenomyosis, together with leiomyoma occurrence, are among the most common benign diseases that occur in the uterus. Although therapy for a leiomyoma is well established except for diffuse leiomyomatosis, no standard treatment for adenomyosis has been determined. As a result, many affected women suffer from physical and psychological symptoms because drug therapy for uterine adenomyosis is limited. Based on these findings, we propose a new adenomyomectomy technique that utilizes a high-frequency surgical loop. With this new technique, we have been able to obtain acceptable results regarding reduced symptoms, such as menstrual pain and excessive menstruation, as well as a high level of patient satisfaction. As for cases of postoperative pregnancy, largely satisfactory results have been obtained with patients under the age of 40 years and 200 births have already been recorded without significant issues. We consider that uterine rupture during a subsequent pregnancy may be avoidable because of improvements in the operative methods. In order to establish this surgical method as an accepted option worldwide, standard treatment protocols under a definitive classification is necessary in parallel with additional research regarding epidemiologic background and the histogenesis of adenomyosis.

\section{References}

[1] G. Younes and T. Tulandi (2018) Conservative surgery for adenomyosis and results: A systematic review. J Minim Invasive Gynecol. 25, 265-276.

[2] H. Takeuchi, M. Kitade, I. Kikuchi, J. Kumakiri, K. Kuroda, and M. Jinushi (2010) Diagnosis, laparoscopic management, and histopathologic findings of juvenile cystic adenomyoma: a review of nine cases. Fertil Steril 94,862-868.

[3] A. Kriplani, R. Mahey, N. Agarwal, N. Bhatla, R. Yadav, and M. Singh (2011) Laparoscopic management of juvenile cystic adenomyoma: four cases. J Minim Invasive Gynecol $18,343-348$.

[4] L. Fedele, S. Bianchi, F. Zanotti, M. Marchini, and G. Candiani (1993) Fertility after conservative surgery for adenomyomas. Hum Reprod 8,1708-1710.

[5] Y. S. Kwon, H. J. Roh, J. W. Ahn, S. H. Lee, and K. S. Im (2015) Conservative adenomyomectomy with transcient occulusion of uterine arteries for diffuse uterine adenomyosis. J Obstet Gynecol Res 41,938-945.

[6] J. K. Kim, C. S. Shin, Y. B. Ko, S. Y. Nam, H. S. Yim, K. and H. Lee (2014) Laparoscopic assisted adenomyomectomy using double flap method. Obstet Gynecol Sci 57,128-135.

[7] A. Saremi, H. Bahrami, P. Salehian, N. Hakak, and A. Pooladi (2014) Treatment of adenomyomectomy in women with severe uterine adenomyosis using a novel technique. Reprod Biomed Online 28,753-760.

[8] Y. S. Kwaon, H. J. Roh, J. W. Ahn, S. H. Lee, and K. S. Im (2013) Laparoscopic adenomyomectomy under transient occlusion of uterine arteries with an endoscopic vascular clip. J Laparoendosc Adv Surg Tech 23,866-870. 
[9] W. M. Liu, C. H.Chen, L. H. Chiu, and C. R. Tzeng (2013) Long-term follow-up of severely symptomatic women with adenomyoma treated with combination therapy. Taiwan $\mathbf{J}$ Obstet Gynecol 52,85-89.

[10] Z. Dai, X. Feng, L. Gao, and M. Huang (2012) Local excision of uterine adenomyomas: a report of 86 cases with follow-up analysis. Eur J Obstet Gynecol Reprod Biol 161, 84-87.

[11] H. Osada, S. Silber, T. Kakinuma, M. Nagaishi, K. Kato, and O. Kato (2011) Surgical procedure to conserve the uterus for future pregnancy in patients suffering from massive adenomyosis. Reprod Biomed Online 22,94-99.

[12] A. Jama FE (2011) Management of adenomyosis in subfertile women and pregnancy outcome. Oman Med J 26,178-181.

[13] A. J. Sun, M. Luo, W. Wang, R. Chen, and J. Lang (2011) Characteristics and efficacy of modified adenomyomectomy in the treatment of uterine adenomyoma. Chin Med J $124,1322-1326$.

[14] Y. S. Kwon, Y. J. Koo, and K. S. Im (2011) Conservative surgical treatment combined with Gn-RH agonist in symptomatic uterine adenomyosis. Pak J Med Sci 27,365-370.

[15] P. H. Wang, W. M. Liu, J. L. Fuh, M. H. Cheng, and H. T. Chao (2009) Comparison of surgery alone and combined surgical-medical treatment in the management of symptomatic uterine adenomyoma. Fertil Steril 92,876-885.

[16] P. H. Wang, J. L. Fuh, H. T. Chao, W. M. Liu, M. H. Cheng, and K. C. Chao (2009) Is the surgical approach beneficial to subfertile women with symptomatic extensive adenomyosis? J Obstet Gynecol Res 35,495-502.

[17] G. Grimbizis, T. Mikos, L. Zepiridis, T. Theodridis, D. Miliaras, B. Tariatzis, and J. Bontis (2008) Laparoscopic excision of uterine adenomyomas. Fertil Steril 89,953-961.

[18] H. Takeuchi, M. Kitade, I. Kikuchi, H. Shimanuki, J. Kumakiri, T. Kitano, and K. Kinoshita (2006) Laparoscopic adenomyomectomy and hysteroplasty: a novel method. J Minim Invasive Gynecol 13,150-154.

[19] C. Wood (1998) Surgical and medical treatment of adenomyosis. Hum Reprod Update 4,150-154.

[20] M. Nishida, K. Takano, Y. Arai, H. Ozone, and R. Ichikawa (2010) Conservative surgical management for diffuse uterine adenomyosis. Fertil Steril 94, 715-719.

[21] A. Fujishita, H. Masuzaki, K. N. Khan, M. Kitajima, and T. Ishimaru (2004) Modified reduction surgery for adenomyosis.
A preliminary report of the transverse $\mathrm{H}$ incision technique. Gynecol Obstet Invest 57,132-138.

[22] S. Preutthipan, and Y. Herabutya (2010) Hysteroscopic rollerball endometrial ablation as an alternative treatment for adenomyosis with menorrhagia and/or dysmenorrhea. J Obstet Gynecol Res 36,1031-1036.

[23] L. Kang, J. Gong, Z. Cheng, H. Dai, and H. Liping (2009) Clinical application and midterm results of laparoscopic partial resection of symptomatic adenomyosis combined with uterine artery occlusion. J Minim Invasive Gynecol 16,169-173.

[24] C. J. Wang, C. F. Yen, C. L Lee, and Y. K. Soong (2002) Laparoscopic uterine artery ligation for treatment of symptomatic adenomyosis. J Am Assoc Gynecol Laparosc 9,293-296.

[25] C. Wood, P. Maher, and D. Hill (1993) Biopsy diagnosis and conservative surgical treatment of adenomyosis. Aust N Z J Obstet Gynecol 33,319-321.

[26] C. Wood, P. Maher, and D. Hill (1994) Biopsy diagnosis and concervative surgical treatment of adenomyosis. J Am Assoc Gynecol Laparosc 1,(4 Pt 1)313-316.

[27] H. Maia, A. Maltez, G. Coelho, C. Athayde, and E. Countinho (2003) Insertion of mirena after endometrial resection in patients with adenomyosis. J Am Assoc Gynecol Laparosc $10,512-516$.

[28] D. Phillips, H. Nathanson, S. Milim, and J. Haselkorn (1996) Laparoscopic bipolar coagulation for the conservative treatment of adenomyomata. J Am Assoc Gynecol Laparosc 4,19-24.

[29] T. Cullen (1908) Adnomyoma of the uterus. Philadelphia, PA: W.B. Saunders

[30] Y. Kishi, H. Suginami, R. Kuramori, M. Yabuta, R. Suginami, and F. Tniguchi (2012) Four subtypes of adenomyosis assessed by magnetic resonance imaging and their specification. Am J Obstet Gynecol 207,114. E1-e7.

[31] M. Nishida, H. Itagaki, Y. Otsubo, R. Ichikawa, Y. Arai, and M. Sakanaka: Histogenesis and classification of diffuse and cystic adenomyosis. J Endometr Pelvic Pain Disord doi: $10.1177 / 2284026518762926$.

[32] M. Nishida, Y. Otsubo, Y. Arai, R. Ichikawa, Y. Kondo, H. Itagaki, and M. Sakanaka (2018) Uterine rupture during subsequent pregnancy following adenomyomectomy - Report of five cases and proposal of prevention.Int J Womens Health Wellness 4,70. doi: 10.23937/2474-1353/1510070. 